\title{
Détection d'une cavité remplie de saumure par imagerie électrique
}

\section{J.-M. MIEHE}

BRGM

3, av. Claude-Guillemin

BP 6009

45060 Orléans

Cedex 02

jm.miehe@brgm.fr

\section{B. FEUGA}

GEORERIS

15. rue Claude-Chappe

BP 95038

57071 Metz Cedex 3

bernard.feuga

@industrie.gouv.fr

C. VACHETTE

GEODERIS

av. de Tsukuba

14209 Hérouville-Saint-Clair

christian.vachette @industrie.gouv.fr
Dans le cadre des problématiques minières liées à I'exploitation du sel et aux vides abandonnés, le BRGM a réalisé une étude géophysique pour GEODERIS sur la concession saline d'Art-sur-Meurthe propriété de la société NOVACARB. L'objectif est d'expérimenter les potentialités de l'imagerie électrique 2D pour la mise en évidence de cavités pleines de saumures. La cavité LR45 de forme circulaire avec un diamètre de $90 \mathrm{~m}$ et une épaisseur de $7 \mathrm{~m}$ se trouve à $115 \mathrm{~m}$ de profondeur. Des modélisations préliminaires ont validé la démarche. Les mesures de résistivité ont mis en évidence une structure conductrice dont les caractéristiques sont en accord avec la géométrie connue de la cavité. Les mesures de polarisation provoquée ont détecté des niveaux polarisants dont l'origine reste à déterminer.

Mots-clés : imagerie électrique 2D, résistivité, chargeabilité, cavité, saumure.

\section{D electrical imaging of a brine filled cavity}

Within the domain of mining problems linked to salt exploitation and abandoned cavities, BRGM has carried out a geophysical study for GEODERIS in the Art-sur-Meurthe brine field which belongs to NOVACARB company. The objective is to test the potential of $2 \mathrm{D}$ electrical imaging so as to focus on salt brine filled cavities. The LR45 cavity, having a circular shape with a $90 \mathrm{~m}$ diameter and a $7 \mathrm{~m}$ thickness, is situated at a depth of $115 \mathrm{~m}$. Preliminary trial runs have given validity to the concept, Resistivity measurements have shed light on a low resistivity structure, of which the characteristics coincide with the established geometry of the cavity. IP measurements detected polarising levels whose origin remains to be determined.

Key words : 2D electrical imaging, resistivity, IP, cavity, salt brine. 


\section{Introduction}

Dans le cadre des problématiques minières liées à l'exploitation du sel et aux vides abandonnés, le BRGM a réalisé pour GEODERIS une étude pour expérimenter les potentialités des méthodes géophysiques dans la mise en évidence de cavités pleines de saumures. Le terrain d'expérimentation a été proposé par la société NOVACARB sur les communes de Lenoncourt et Artsur-Meurthe. Il s'agit de la concession saline d'Art-surMeurthe où les cavités sont bien connues en profondeur et géométrie, En particulier, la cavité LR45 a été auscultêe par sonar (SOCON) et pour cette raison a été retenue pour les investigations géophysiques. Les modélisations préliminaires ont montré que l'imagerie électrique 2D est une technique adaptée à cette problématique. La méthode retenue est celle du panneau électrique en dispositif dipôle-dipôle de longueurs $D=50$ et $100 \mathrm{~m}$ avec mesure simultanée de la résistivité apparente et de la polarisation provoquée.

\section{2}

\section{Principe du panneau électrique en dispositif dipôle-dipôle}

La méthode électrique consiste à injecter un courant électrique d'intensité I entre deux électrodes A et B (dipôle émetteur) et à mesurer la différence de potentiel résultante $V$ entre deux électrodes $M$ et $N$ (dipóle récepteur). L'ensemble émetteur-récepteur constitue un quadripôle. Les grandeurs mesurées sont les suivantes :

- la résistivité apparente, définie comme étant la résistivité du sol homogène et isotrope qui, avec le quadripôle utilisé, donnerait entre $\mathrm{M}$ et $\mathrm{N}$ la différence de potentiel V;

- la polarisation provoquée (PP) ou chargeabilité. Lorsqu'on envoie dans le sol un courant entre A et B et que l'on coupe brutalement ce courant, la différence de potentiel entre $\mathrm{M}$ et $\mathrm{N}$ ne chute pas instantanément mais nécessite quelques centaines de millisecondes à quelques secondes pour se dissiper. La polarisation provoquée caractérise l'aptitude du sol à conserver une charge de courant pendant un temps très court (effet capacitif).

Ces deux paramètres sont enregistrés simultanément et dépendent des propriétés électriques du sol et de la géométrie du dispositif utilisé. La résistivité apparente permet de mettre en évidence les structures présentant un contraste de résistivité électrique. La chargeabilité a été essentiellement appliquée à la recherche de minéralisations à sulfures disséminées ou de ressources en eau et plus récemment à la détection de pollutions par hydrocarbures. La méthode du panneau électrique utilise un ensemble d'électrodes régulièrement espacées le long d'un profil rectiligne. Les grandeurs caractéristiques du dispositif dipôle-dipóle (Fig. 1) sont la dimension des dipôles $A B$ et $M N$ de longueur $D$ et le rang $n$, grandeur entière qui indique la distance exprimée en longueurs de dipôles entre la partie émettrice $\mathrm{AB}$ et la partie réceptrice $\mathrm{MN}$ du dispositif.

Pour une position donnée de l'émetteur AB, les mesures sont effectuées en augmentant progressivement la distance entre $\mathrm{AB}$ et MN. La séquence est répétée pour toutes les positions du dipôle $A B$ le long du profil. Les mesures sont conventionnellement représentées sous forme de pseudo-coupes de résistivité apparente ou de chargeabilité en fonction du rang $\mathrm{n}$. La position d'une mesure donnée est celle du milieu du segment émetteur-récepteur sur la bissectrice à $45^{\circ}$. Le paramètre $n$, indicateur de la dimension du dispositif d'électrodes, ne constitue pas une vraie échelle de profondeur. Les pseudo-coupes sont généralement interprétées par calcul direct ou par inversion. L'interprétation permet d'établir une coupe de résistivité vraie en fonction de la profondeur directement interprétable en termes de structures géologiques.

\section{3}

\section{Modélisations préliminaires}

La cavité LR45 est de forme cylindrique avec un diamètre de $90 \mathrm{~m}$ et une hauteur de $7 \mathrm{~m}$. Le toit de la cavité

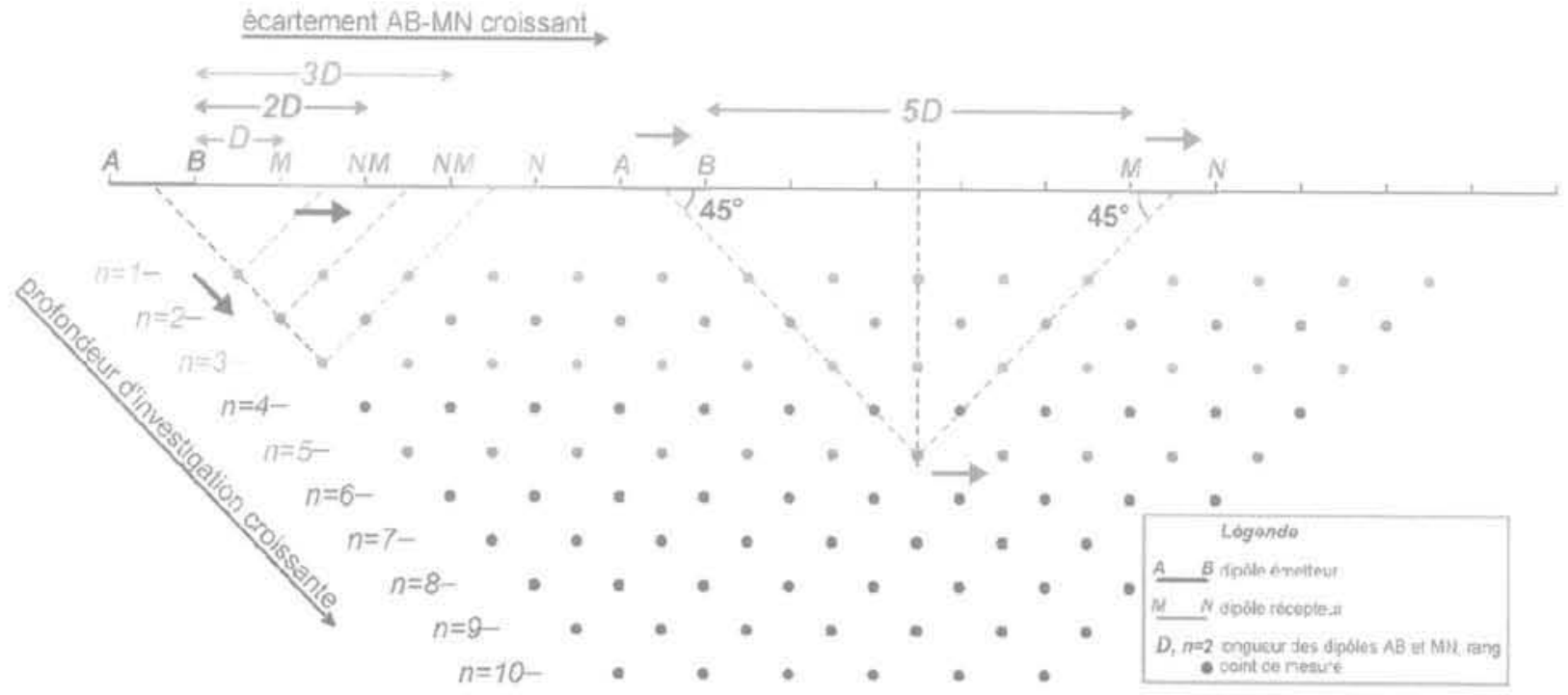

FIG.1 Principe de la mesure en dispositif dipôle-dipôle. Principle of measurement using a dipole-dipole array. 


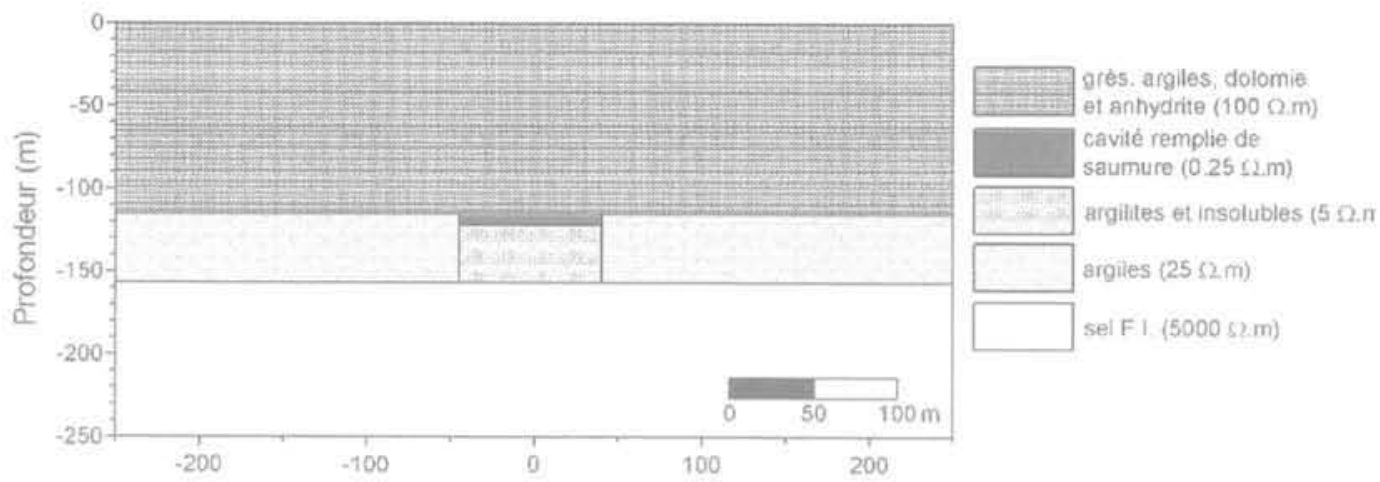

FiG. 2 Modèle géoélectrique simulé. Simulated electrical model.

remplie de saumure est à $115 \mathrm{~m}$ de profondeur. Au toit. la cavité est surmontée d'un ensemble grès/argile/dolomie/anhydrite. L'encaissant est constitué d'argiles, le toit du sel est à environ $35 \mathrm{~m}$ sous la base de la cavité, constituée d'une cavité remplie d'insolubles imbibés d'eau salée. Le paramètre déterminant est la résistivité apparente. Un modèle géoélectrique a été défini à partir de la géométrie connue de la cavité et d'hypothèses réalistes de résistivité des différentes formations en présence (Fig. 2). L'eau salée (< $1 \Omega . \mathrm{m}$ ) présente un très fort contraste de résistivité avec les formations géologiques environnantes (ensemble grès/argile/dolomie/anhydrite environ $100 \Omega$.m, argiles 10 à $50 \Omega$.m, sel $>5000 \Omega . m$ ). La réponse de ce modèle a été calculée par simulation d'un dispositif dipôle-dipôle $\mathrm{D}=100 \mathrm{~m}$. Le résultat de la simulation est une pseudo-coupe de résistivité apparente (Fig. 3a) qui met en évidence une anomalie conductrice (environ $60 \Omega$.m) à l'aplomb des cavités remplies de saumure et d'insolubles, dans un contexte de 90 à $180 \Omega$.m. Les figures $3 \mathrm{~b}$ et $3 \mathrm{c}$ montrent, avec les hypothèses de résistivité adoptées, que la contribution de la cavité remplie d'insolubles est prépondérante devant l'effet de la cavité remplie de sau. mure. Même en intégrant le bruit géologique (variations d'épaisseur ou de résistivité des formations), les contrastes de résistivité sont suffïsants pour être mesurés de façon fiable.

La pseudo-coupe simulée (Fig. 3a) a été inversée avec le logiciel RES2DINV (M.H. Loke). L'exercice a pour but de comparer le modèle géoélectrique de départ avec la coupe de résistivité inversée, pour évaluer les performances du processus d'inversion. (a) effets des cavités remplies de saumure et d'insolubles $900-800-700-500 \cdot 500-400-300-200 \cdot 100 \quad 0 \quad 100 \quad 200 \quad 300 \quad 400 \quad 500 \quad 600 \quad 700 \quad 800 \quad 900$

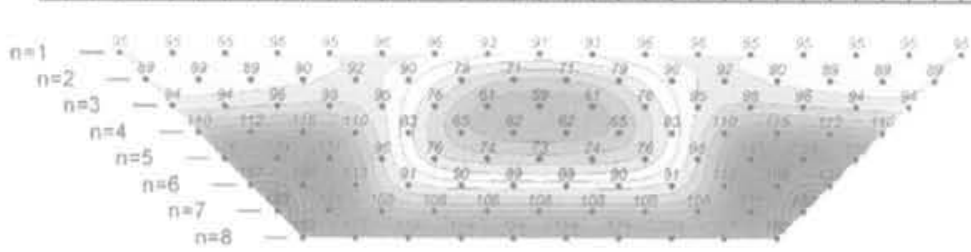

(b) effets de la cavite remplie d'insolubles seufe

$-900-800-700-600-500-400-300-200-100 \quad 0 \quad 100 \quad 200 \quad 300 \quad 400 \quad 500 \quad 600 \quad 700 \quad 800 \quad 900$

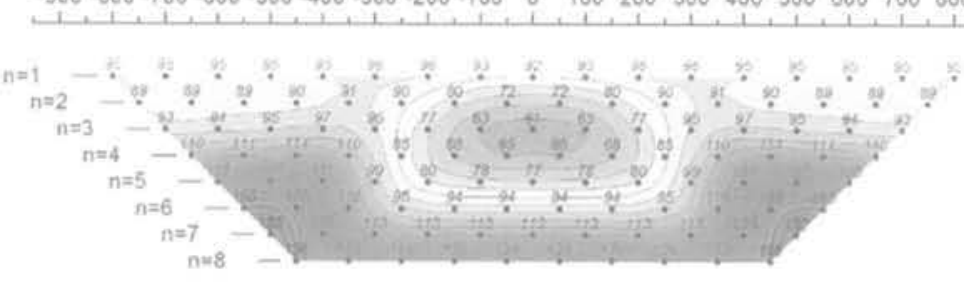

(c) effets de la cavite remplie de saumure seufe

$-900-800-700-600-500-400-300-200-100 \quad$ D $\quad 100200 \quad 300 \quad 400 \quad 500 \quad 600 \quad 700 \quad 800 \quad 900$

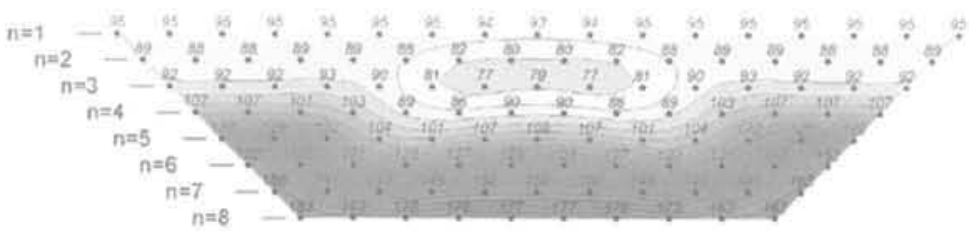

Résistivité apparente

$(\Omega, \mathrm{m})$

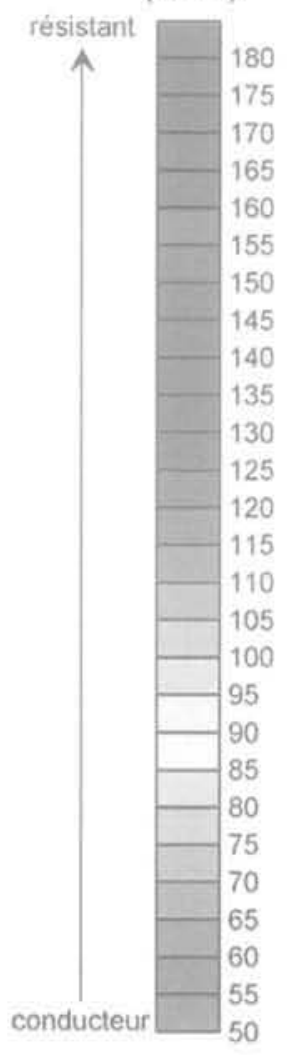

FIG. 3 Modélisation 2D de la cavité LR45, pseudo-coupes de résistivité apparente. 2D modelisation of LR45 cavity, apparent resistivity pseudo-sections. 

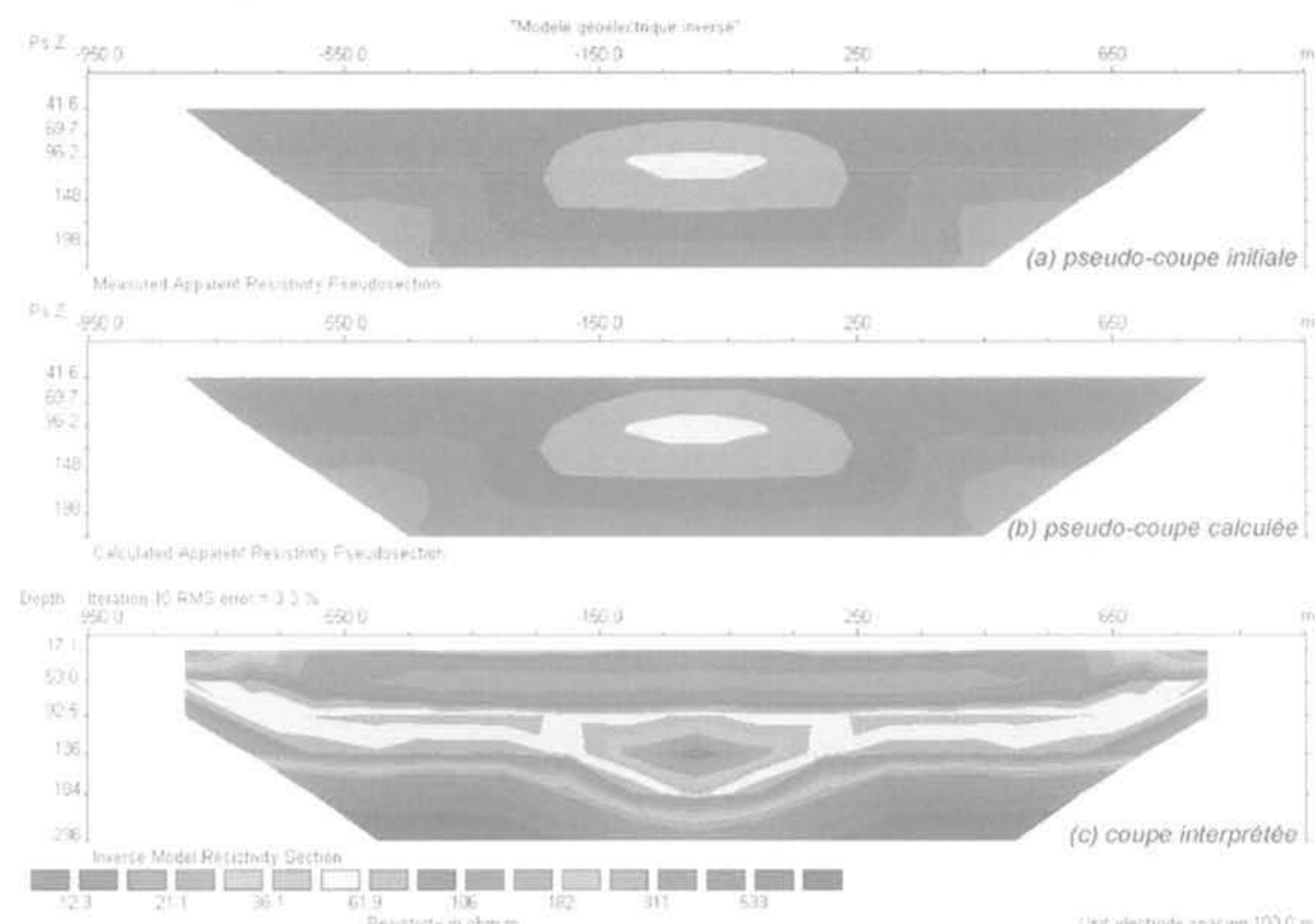

FIG.4 Inversion de la pseudo-coupe modélisée.

Inversion of simulated pseudo-section.

Les résultats de cette opération sont présentés sur la figure 4. La coupe (a) est la pseudo-coupe de résistivité apparente simulée par calcul direct, la coupe (b) est la coupe de résistivité apparente calculée à partir de la distribution de résistivité (c) résultat de l'inversion. Le processus d'inversion minimise l'écart quadratique moyen (RMS) entre les pseudo-coupes (a) et (b). En ce sens l'interprétation est satisfaisante puisque les deux coupes sont très semblables et l'écart quadratique moyen (RMS) est faible $(3,3 \%)$.

La coupe inversée (Figs $4 c$ et 5) met bien en évidence une structure conductrice centrée et calée en profondeur sur les cavités. L'inversion ne permet cependant pas de distinguer la cavité remplie de saumure de la cavité remplie d'insolubles, ce résultat était prévisible d'après les modélisations par calcul direct.

La coalescence avec les argiles conductrices ne permet pas de délimiter les cavités de façon précise le long du profil. Les artefacts liés à l'inversion font que le toit des argiles conductrices est mal placé alors que le toit de la formation salifère résistante est nettement mieux défini. Les contrastes de résistivité sont conservés et suffisamment marqués pour caractériser les différentes unités géologiques même si en valeur absolue on ne retrouve pas les résistivités du modèle géoélectrique simulé

\section{4}

\section{Acquisition des données}

Le panneau électrique est centré sur le forage LR45 et couvre une longueur de $1600 \mathrm{~m}$ le long d'un profil orienté $N^{\circ} 69$ (Fig. 6).

Le dispositif dipôle-dipôle combine deux longueurs de dipôle, $\mathrm{D}=50 \mathrm{~m}$ et $100 \mathrm{~m}$ pour assurer une définition homogène aux différentes profondeurs et pour atteindre une profondeur d'investigation maxi-

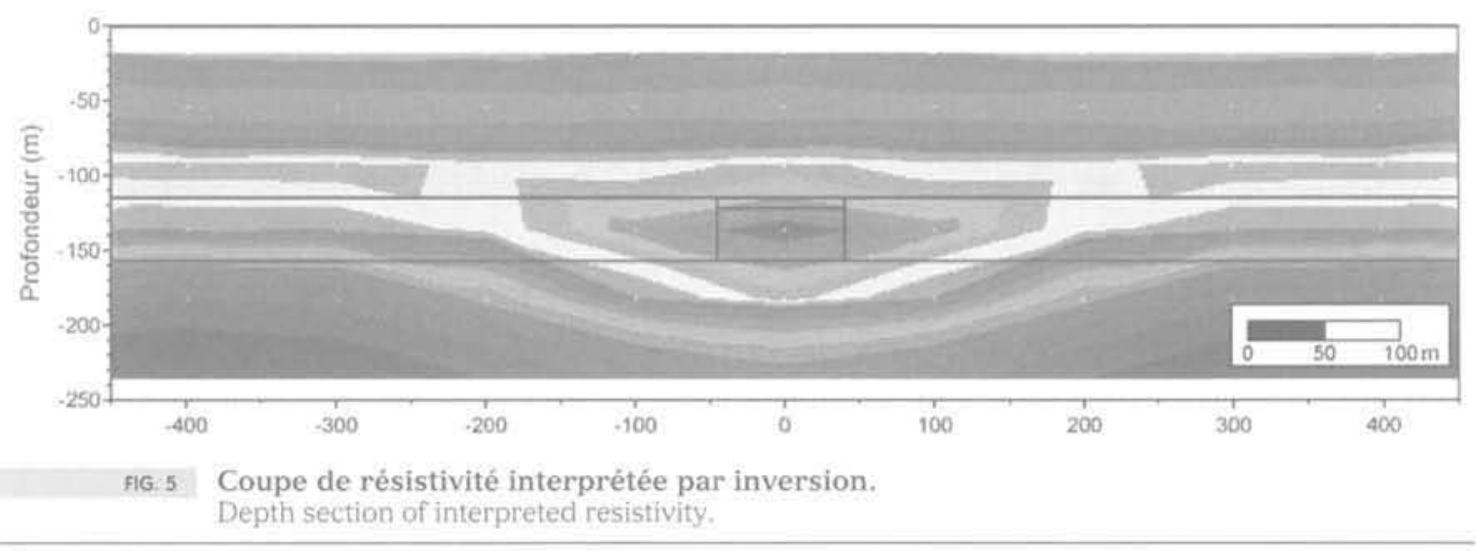




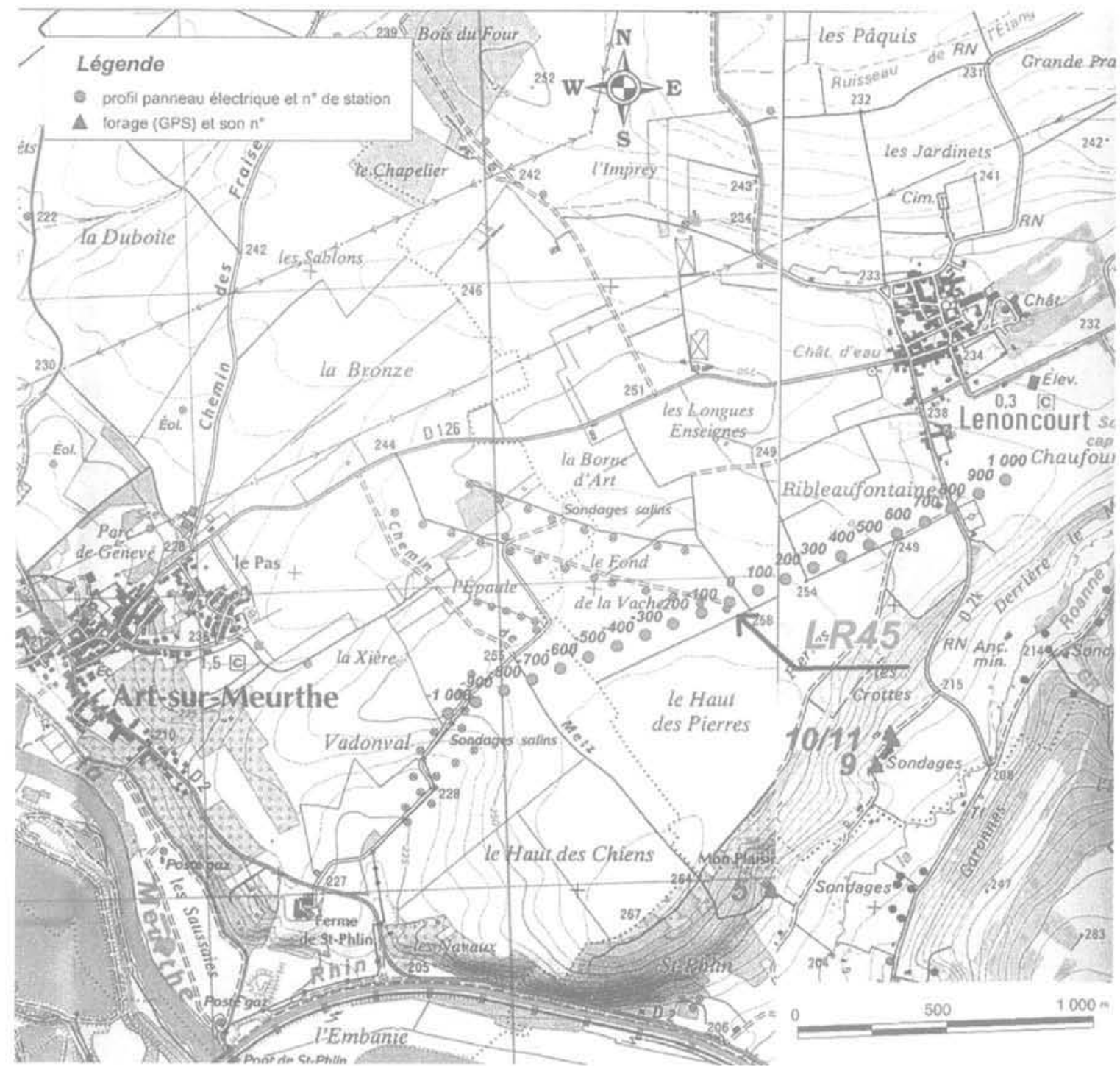

FG.6 Localisation du panneau électrique (extraits des cartes IGN 3415 Est

Saint-Nícolas-de-Port et 3315 Est Nancy-Toul).

Location of the study area.

male d'environ $180 \mathrm{~m}$. La topographie a été mesurée par nivellement direct pour prendre en compte les variations de relief. Les statistiques relatives aux mesures de résistivité apparente sont résumées dans le tableau I.

Sur l'ensemble du panneau mesuré, les écarts constatés sur les mesures répétées n'excèdent pas $0,5 \mathrm{mV} / \mathrm{V}$ en polarisation provoquée et quelques dixièmes d' $\Omega$.m en résistivité apparente. Les pseudocoupes intègrent des artefacts liés, d'une part, au mode de représentation et à la géométrie des dispositifs électriques (chapeaux chinois, branches inclinées à $45^{\circ}$ ) et. d'autre part, les effets des à-coups de prise produits par les hétérogénéités superficielles. Les pseudo-coupes ont été interprétées par inversion. Le logiciel permet de calculer et de corriger l'effet du relief topographique. le modèle peut être contraint par des informations a priori (résistivité vraie des formations, profondeur des interfaces, structures horizontales, verticales ou inclinées).

TABLEAUI Statistiques relatives au panneau électrique. Statistics on electrical measurement.

\begin{tabular}{c|c|c|c|c}
\hline Dispositif & $\begin{array}{c}\text { Nombre } \\
\text { de mesures }\end{array}$ & $\begin{array}{c}\text { Nombre } \\
\text { de lignes }\end{array}$ & $\begin{array}{c}\text { Valeur minimale } \\
\text { de résistivité apparente } \\
(\Omega . m) \text { et de } \\
\text { chargeabilité (mVN) }\end{array}$ & $\begin{array}{c}\text { Valeur maximale } \\
\text { de résistivité apparente } \\
(\Omega \mathrm{m}) \text { et de } \\
\text { chargeabilité (mVN) }\end{array}$ \\
\hline $\mathrm{D}=50 \mathrm{~m}$ & 120 & $\mathrm{n}=1 \mathrm{à} 4$ & $14 / 0,8$ & $56 / 8,6$ \\
\hline $\mathrm{D}=100 \mathrm{~m}$ & 113 & $\mathrm{n}=1$ à 8 & $19 / 0,7$ & $96,2 / 6,5$ \\
\hline
\end{tabular}


5

\section{Résultats - discussion}

Les pseudo-coupes de résistivité apparente et de polarisation provoquée sont représentées sur la figure 7 .
Les coupes de résistivité et de polarisation provoquée interprétées sont représentées sur la figure 8. La coupe de résistivité met en évidence plusieurs unités géoélectriques. La structure conductrice C1 est conforme au modèle géoélectrique connu. Les cavités remplies de saumures et d'insolubles sont clairement localisées sur l'emprise du conducteur C1. La zone
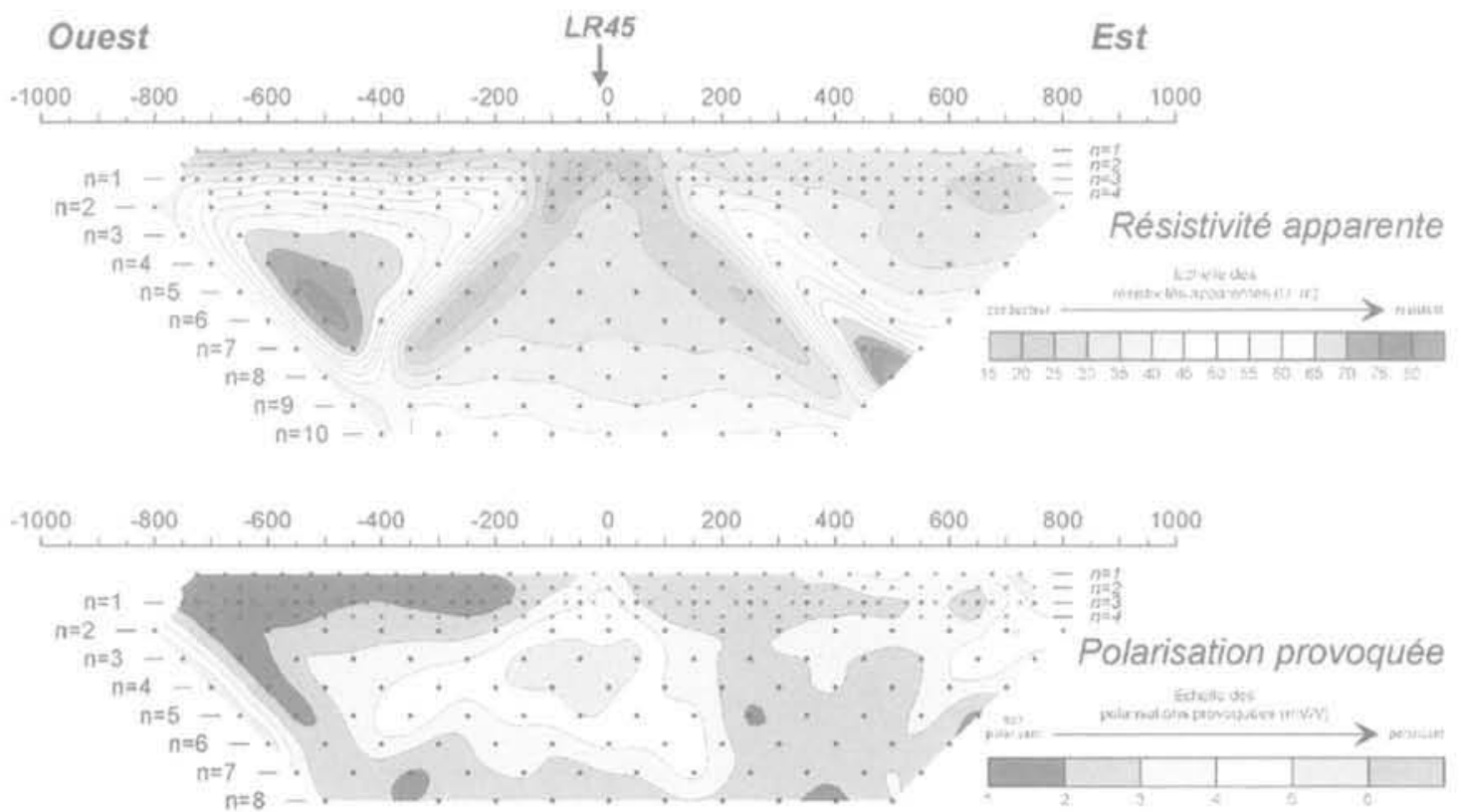

FiG.7 Pseudo-coupes de résistivité apparente et de polarisation provoquée. Apparent resistivity and IP pseudo-sections.
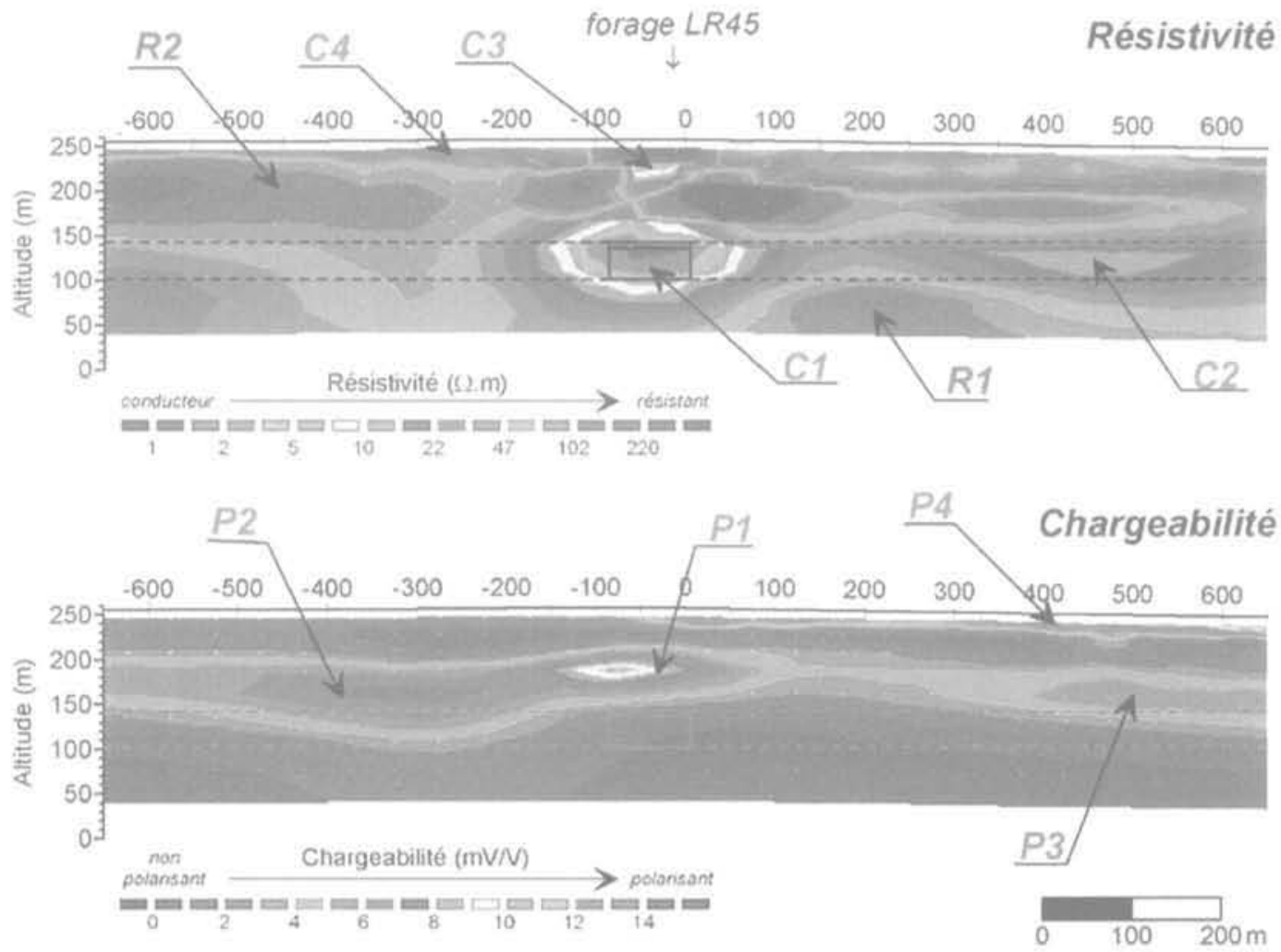

FiG.8 Coupes de résistivité et de chargeabilité interprétées. Interpreted resistivity and IP sections. 
d'amplitude minimale $(<1 \Omega \mathrm{m})$ est proche de la cavité remplie de saumure. Vers l'est le conducteur C2 (10 à $30 \Omega, \mathrm{m}$ ) coincide avec les argiles et surmonte un ensemble résistant $\mathrm{R} 1$ (>300 $\Omega$.m) dont le toit correspond à celui de la formation salifère (altitude $100 \mathrm{~m}$ ).

Vers l'ouest le schéma est plus complexe, la formation salifère (altitudes inférieures à $100 \mathrm{~m}$ ) apparait plus conductrice ( 20 à $70 \Omega$.m). Les argiles semblent céder la place à un ensemble plus résistant et subvertical entre les stations -450 et - 200. La structure résistante R2 (70 à $150 \Omega . m$ ) correspond à l'ensemble grès/argiles/dolomie/anhydrite. Ce résistant se poursuit tout le long du profil, très homogène vers l'ouest il semble marqué par des alternances plus argileuses vers l'est et est interrompu entre les stations -50 et 0 par une structure très conductrice C 3 (<10 $\Omega$ m). Ce conducteur n'est pas directement lié au forage (fuite d'eau salée), les contrôles effectués par NOVACARB dans le forage L.R45 n'ont détecté aucune anomalie dans la structure du forage: L'origine de ce conducteur pourrait être liée à l'existence d'une faille dans le rhétien situé à proximité du forage LR45, avec des remontées de saumures le long de cette faille, suivant un mécanisme qui reste à déterminer.

Le conducteur C4 (20 $\Omega . \mathrm{m}$ ) entre les stations - 650 et -100 est probablement dû à une composante plus limoneuse des terrains les plus superficiels.

La coupe de polarisation provoquée montre un contexte peu polarisant inférieur à $3 \mathrm{mV} / \mathrm{V}$ et met en évidence deux horizons polarisants. Le premier ensemble est situé au toit des argiles. D'une épaisseur relativement homogène d'environ $50 \mathrm{~m}$ ce polarisant couvre le profil d'est en ouest et regroupe les structures
P1, P2 et P3 avec des chargeabilités comprises entre 4 et $12 \mathrm{mV} / \mathrm{V}$. L'amplitude maximale est relevée au-dessus de la cavité remplie de saumure (P1, $12 \mathrm{mV} / \mathrm{V})$, entre les deux conducteurs C1 et C3, sans que l'on puisse établir un lien avec la cavité. Le second ensemble polarisant est superficiel et peu enraciné (P4, 4 à $8 \mathrm{mV} / \mathrm{V}$ ), entre les stations 0 et 650 . L'origine exacte des polarisants reste à déterminer.

\section{6}

\section{Conclusion}

Le panneau électrique a mis en évidence une structure conductrice conforme au modèle géoélectrique connu. Les cavités remplies de saumure et d'insolubles sont clairement localisées sur l'emprise du conducteur. Un second conducteur, moins profond et moins étendu, a été détecté autour du forage. Il pourrait s'agir d'une remontée de saumure le long d'une faille observé au voisinage du forage LR45 dans le rhétien. La coupe de polarisation provoquée montre deux ensembles polarisants dont l'origine reste à déterminer. Le lien entre la structure polarisante localisée audessus des cavités et les cavités reste à démontrer. Ces mesures permettent d'identifier et de localiser sans. ambiguité les cavités remplies de saumure et d'insolubles et valident complètement la technique du panneau électrique mais montrent aussi ses limites: les dimensions en regard de la profondeur ne produisent pas un contraste de résistivité suffisant pour séparer les effets de la cavité remplie de saumure des effets de la cavité remplie d'insolubles.

\section{Bibliographie}

Cahyna F.. Mazac O., Venhodova D. - Determination of the extend of cyanic contamination by surface geoelectrical methods, In Geotechnical and Fnvironmental Geophysics, Ward S.H. (ed.), vol. 2, Environmental and Groundwater. Society of Exploration Geophysicists. Tulsa, 1990, p. $97-99$.

Dam (van) J.C. Meulenkamp J.J. - Some results of the geo-electrical resistivity method in ground water investigations in the Netherlands. Geophysical prospecting, 15 (1), 1967, p. 92-115.

Edwards L.S, - A modified pseudosection for resistivity and induced-polarization. Geophysics, 42, 1977, p. 1020-1036.

Gréau E. - Le sel en Lorraine. BergerLevrault et Cie éditeurs, 1908.

Ghoreychi M. Daupley X. - Devenir à long terme d'exploitations abandonnées de sel. Colloque Après-mine, Nancy, 57 février 2003.

Keller G.V., Frischknecht F.C. - Electrical methods in geophysical exploration. Pergamon, New York, 1966, $519 \mathrm{p}$.
Loke M.H. Barker R.D. - Rapid leastsquares inversion of apparent resistivity pseudosections by a quasi-Newton method. Geophysical Prospecting, 44. 1996, p. 131-152.

Loke M.H., Barker R.D. - Practical techniques for 3D resistivity surveys and data inversion. Geophysical Prospecting. 44, 1996, p. 499-523.

Marchal C. - Le gite salifère keupérien de Lorraine-Champagne et les formations associées - Etude géométrique - Implications génétiques. Thèse de $3^{8}$ cycle, Université Nancy I, 30 juin 1982.

Maubeuge P.L. - Le bassin salifère lorrain. These, Imprimerie Georges-Thomas, Nancy, 1950.

Ogilvy A.A., Kuzmina E.N. - Hydrogeologic and engineering geologic possibilities for employing the method of induced potentials, Geophysics, 37 (5). 1972, p. 839-861.

Reynolds J.M. - An introduction to applied and environmental geophysics. John Wiley \& Sons Ltd., England, 1997, 796 p.
Summer J.S, - Principles of induced polarization for geophysical exploration. Elsevier Scientific Publishing Company, 1976.

Tweeton D.R., Cumeriato C.L., Jay C., Kuhlman H.H.L - Field test of geophysical techniques for predicting and monitoring leach solution flow during in situ mining. Geoexploration, 28, 1991. p. $251-268$.

Vacqujer V.. Holmes C.R. Kintzincer P.P. Lavergne M. - Prospecting for groundwater by induced electrical polarization. Geophysics, 22 (3), 1957, 660-687.

Ward S.H. - Resistivity and induced polarization methods. In Geotehnical and Environmental Geophysics, Ward, S.H. (ed.), Society of Exploration Geophysicists, Tulsa, 1990, p. 147-149.

Yaramanci U. - Geoelectric exploration and monitoring in rock salt for the safety assessment of underground waste disposal sites. Journal of Applied geophysics, 44,2000 , p. 181196. 